Revista PSICOLOGIA, 2014, Vol. 28 (2), 1-10

\title{
A versão portuguesa do NEO-FFI: Caracterização em função da idade, género e escolaridade
}

\author{
Margarida Pedroso-Lima ${ }^{1}$, Eunice Magalhães ${ }^{2}$, Ana Salgueira ${ }^{2}$, António-José Gonzalez ${ }^{3}$, \\ José Joaquim Costa ${ }^{1}$, Manuel João Costa ${ }^{2}$, Patrício Costa ${ }^{2}$ \\ ${ }^{1}$ Faculdade de Psicologia e de Ciências da Educação, Universidade de Coimbra \\ ${ }^{2}$ Escola das Ciências da Saúde, Universidade do Minho \\ ${ }^{3}$ Instituto de Psicologia Aplicada, ISPA - University Institute
}

Resumo: 0 presente estudo tem como objetivo apresentar dados descritivos e multivariados obtidos com o NEO-FFI, uma versão reduzida do NEO-PI-R, com uma amostra de participantes portugueses. Para tornar possível este objetivo, o inventário de personalidade foi administrado a 1178 sujeitos, $68 \%$ do sexo feminino, e com idades compreendidas entre os 18 e os 90 anos $(M=41.8, D P=22.3)$. A versão portuguesa do NEO-FFI reflete a universalidade das dimensões básicas da personalidade podendo, por conseguinte, ser usada como uma versão fiável do NEO-PI-R. Estes dados reforçam os resultados obtidos internacionalmente, e serão discutidos no presente artigo à luz da literatura sobre o modelo dos cinco fatores. Os resultados revelam ainda um efeito significativo do sexo e da escolaridade ao nível da personalidade (controlando a variável idade), especificamente, no que diz respeito às dimensões de Abertura à Experiência, Neuroticismo, Conscienciosidade e Amabilidade. No que se refere à Extroversão não foram encontrados efeitos significativos das variáveis individuais referidas. Verificámos ainda que à medida que a idade avança diminuem as pontuações de Extroversão e de Abertura à Experiência.

Palavras-chave: Personalidade; NEO-FFI; Modelo dos Cinco Fatores.

The Portuguese version of the NEO-FFI: age, gender and education characterization: The present study aims to present descriptive and multivariate data obtained with the NEO-FFI, a reduced version of the NEO-PI-R, with a sample of Portuguese participants. To make possible this objective, the personality inventory was administered to 1178 subjects, $68 \%$ were female, with ages between 18 and 90 years $(M=$ $41.8, S D=22.3$ ). The Portuguese version of the NEO-FFI reflects the universality of basic dimensions of personality and may, therefore, be used as a reliable version of the NEO-PI-R. These data reinforce the results obtained internationally, and will be discussed in this article at the light of the literature about the five factor model. The results also reveal a significant effect of gender and educational level on personality (controlling age), specifically with regard to the dimensions of Openness, Neuroticism, Conscientiousness and Agreeableness. Concerning Extroversion this significant effects were not found. We also encountered that, with age, the scores in Extroversion and Openness tend to get lower.

Keywords: Personality; NEO-FFI; Five Factor Model.

O modelo dos cinco fatores (Five Factor Model: FFM; McCrae \& Costa, 2004) envolve uma representação dimensional das diferenças interpessoais ao nível da personalidade cuja validade, compreensividade, universalidade, hereditabilidade e estabilidade longitudinal tem sido recorrentemente sublinhada pela investigação empírica (McAdams \& Pals, 2006). Através deste modelo, é possível aglutinar as tendências comportamentais, emocionais e cognitivas das pessoas em cinco grandes categorias: Neuroticismo (N), Extroversão (E), Abertura à Experiência (O), Amabilidade (A) e Conscienciosidade (C). Estas dimensões têm sido replicadas em auto e hetero-avaliações, em diferentes línguas e culturas e, com amostras clínicas e normativas (McCrae, Costa, del Pilar, Rolland, \& Parker, 1998; McCrae \& Terracciano, 2005). Estes dados fundamentam o consenso substancial em torno da estrutura dos traços da personalidade na idade adulta e consagram o modelo como uma ferramenta útil para descrever a personalidade do adulto e para guiar a investigação (Rolland, Parker, \& Stumpf, 1998).

0 modelo tem sido operacionalizado através do NEO-PI-R, que avalia estas cinco principais dimensões, ou domínios, da personalidade, assim como um conjunto de facetas ou traços que definem

Dados de contacto para correspondência: Margarida Pedroso de Lima, Faculdade de Psicologia e de Ciências da Educação, Universidade de Coimbra, Rua Colégio Novo, 3000 Coimbra, Portugal. E-mail: mplima@fpce.uc.pt. 
cada um dos referidos domínios. Em conjunto, os cinco domínios (OCEAN) ${ }^{2}$ e as trinta escalas das facetas permitem uma avaliação compreensiva da personalidade adulta. Este instrumento elaborado por Costa e McCrae (1992) constitui a última versão da revisão do NEO-PI - o primeiro instrumento a operacionalizar o modelo FFM - que começou a ser construído em 1978 através de uma combinação de métodos teóricos e de análise fatorial.

No que diz respeito à versão portuguesa (Lima \& Simões, 1997), foi obtida uma estrutura fatorial congruente com o Modelo dos Cinco Fatores, explicando $21 \%{ }^{3}$ da variância, semelhante ao obtido na versão Americana - 23\% da variância (Costa, McCrae \& Dye, 1991). Além disso, os coeficientes de consistência interna (Alfas de Cronbach) dos fatores com uma amplitude situada entre .80 para a Extroversão e .86 para a Conscienciosidade são considerados "bons"4. Na versão original (Costa, McCrae, \& Dye, 1991), bem como, na portuguesa, as cinco dimensões da personalidade foram associadas a uma grande diversidade de variáveis psicológicas e demográficas (e.g., bem-estar, género) encontrando-se variações relacionadas com a idade, profissão, educação e género (Lima, 1997).

O NEO-PI-R apresenta, por conseguinte, inúmeras vantagens derivadas da extensa investigação (McAdams \& Olson, 2010), em que se baseia, da acesa discussão científica que tem gerado, por adiantar uma proposta de análise compreensiva da personalidade, que representa uma tentativa de dar sentido à multiplicidade de constructos existentes, no domínio da psicologia da personalidade (McCrae \& Costa, 2003, 2008). Efetivamente, uma grande diversidade de constructos é abarcada na sua interpretação (e.g., bem-estar, resiliência, empenhamento e criticismo; McAdams \& Olson, 2010); uma vasta literatura permitiu compará-lo com dezenas de outros modelos, constructos e instrumentos de personalidade (e.g., EPQ, PFR, 16PF, MBTI, CPI e MMPI; Little \& Joseph, 2007); e as suas qualidades psicométricas foram comprovadas em muitas populações, de diversos países (McCrae \& Costa, 2004). Uma outra vantagem é o facto de se tratar de um instrumento especificamente construído para adultos ${ }^{5}$ : pode aplicar-se, durante toda a idade adulta (a partir dos 17 anos), a sujeitos de quase todos os níveis de escolaridade e proveniência social e em contextos díspares, como as áreas do aconselhamento, psicologia clínica, psicologia da saúde, psiquiatria, psicologia educacional, orientação escolar e profissional, psicologia organizacional e investigação (Costa \& McCrae,1992). Além disso, é um instrumento simples de administrar, cotar e interpretar por profissionais com formação em Psicologia e em avaliação da personalidade, proporciona auto e hetero-avaliações (formas S e R do inventário) e permite fornecer feedback ao sujeito. Em suma, o modelo dos cinco fatores oferece um guia conceptual, que pode ser utilizado, sempre que é avaliada a personalidade.

Apesar das inúmeras vantagens deste instrumento, a sua extensão - 240 itens - tornam-no moroso e pouco versátil. Esta limitação levou à construção de versões reduzidas deste instrumento, tipicamente designadas por NEO-FFI ou NEO-FFI-R (na versão revista) ${ }^{6}$. O NEO-FFI (NEO-Five Factor Inventory; Costa \& McCrae, 1989) é assim uma versão reduzida do NEO-PI-R, constituída por 60 itens e que, no original, nos permite obter uma versão fiável dos domínios do modelo dos cinco fatores (com valores de consistência interna entre .68 e .86; Costa \& McCrae, 1989). Várias adaptações têm sido desenvolvidas em diferentes países (cf. Anexo 1), sendo os valores de consistência interna entre culturas genericamente aceitáveis e similares, refletindo o potencial do presente instrumento para ser utilizado em diferentes contextos. 0 NEO-FFI tem também sido utilizado extensivamente para estudar as relações entre as cinco dimensões da personalidade e outros constructos como, por exemplo, estilos de aprendizagem (Chamorro-Premuziac \& Furnham, 2009), problemas interpessoais (Nyater, Langvik, Berthelsen, \& Nordvik, 2009), perturbações da personalidade (Aluja Garcia, Cuevas, \& Garcia, 2007) ou sucesso académico (Chamorro-Premuzic, Quiroga \& Colom, 2009).

As limitações identificadas nas adaptações do NEO-FFI passam pelas dificuldades na reprodução da sua estrutura original nalguns países e a sua suscetibilidade ao engano deliberado (Furham, 1997; Korner, Geyer, Roth, et al., 2008; Panayiotou, Kokkinosb, \& Spanoudisa, 2004). McCrae e Costa (2004) fizeram uma revisão ao NEO-FFI original, substituindo 14 itens por itens do NEO-PI-R, de acordo com os seguintes critérios: i. minimizar dos efeitos da aquiescência; ii. aumentar as correlações com as pontuações dos fatores do NEO-PI-R; iii. diversificar o conteúdo dos itens selecionando itens de facetas

\footnotetext{
2 Podemos sintetizar o significado dos domínios, através das seguintes dicotomias: Extroversão (retraído-sociável; cautelosoaventureiro); Neuroticismo (calmo-ansioso; seguro-inseguro); Amabilidade (antagonista-generoso); Conscienciosidade (irresponsável-responsável; desorganizado-organizado); Abertura à Experiência (convencional-original).

${ }^{3}$ A percentagem reduzida da variância dos resultados é algo conhecido nos modelos fatoriais da personalidade e significa que apesar da tendência para nos comportarmos de uma determinada maneira, fruto da nossa constelação de traços, há muitas outras variáveis que afetam a forma como nos vamos comportar/sentir numa determinada situação/momento.

${ }^{4}$ Cf. e.g., EFPA review model for the description and evaluation of Psychological and Educational Tests (2013).

${ }^{5}$ No entanto, em 2005 começaram a surgir versões (NeO-PI-3; McCrae, Costa, \& Martin, 2005) aplicáveis a indivíduos com idades inferiores a 17 anos.

${ }^{6}$ Múltiplas outras versões reduzidas têm sido propostas, a título de exemplo: NEO-60 (Aluja, Garcia, Cuevas, \& Garcia, 2005).
} 
sub-representadas e aumentar a compreensibilidade dos itens. A validade e a fiabilidade da nova versão tem vindo a ser demonstrada (Alfas de Cronbach entre 75 e .82) (McCrae \& Costa, 2004). No contexto português, Barros (1979) utiliza pela primeira vez uma versão reduzida, Bertoquini e Pais Ribeiro (2006) apresentam estudos com uma versão do NEO-FFI, do NEO-FFI-R e do NEO-FFI-P, Lima (2008) com o NEOFFI e, mais recentemente, Magalhães e colaboradores (2014), a partir da versão validada do NEO-PI-R, testam a validade de constructo do NEO-FFI através da Análise de Componentes Principais (ACP) com rotação Varimax e da Análise Fatorial Confirmatória (AFC) com recurso a modelos unifatoriais, usando o método da máxima verosimilhança (Maximum Likelihood) para cada dimensão da personalidade em separado. Os cinco fatores de personalidade foram extraídos através da ACP, explicando $35.1 \%$ da variância, tendo sido confirmados posteriormente na AFC que revelou índices de ajustamento adequados. No que diz respeito à consistência interna, foram obtidos valores entre .69 (Abertura à Experiência) e .81 (Conscienciosidade), considerando-se aceitáveis e comparáveis com os da versão original (Magalhães et al., 2014). No sentido de providenciar dados adicionais que permitam a comparabilidade de resultados aquando da utilização do NEO-FFI no contexto português, serão descritos no presente estudo dados descritivos das pontuações obtidas no NEO-FFI numa amostra de sujeitos com características individuais diferenciadoras, e a sua relação com variáveis sociodemográficas dos participantes.

\section{MÉTODO}

\section{Participantes}

Participaram no presente estudo 1178 sujeitos, na sua maioria do sexo feminino ( $\mathrm{N}=801)$, e com idades compreendidas entre os 18 e os 90 anos $(M=41.8, D P=22.3)$. Em termos de escolaridade, $38 \%$ concluiu

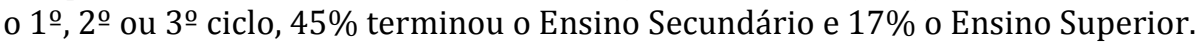

\section{Instrumentos}

Foi utilizada a versão Portuguesa do NEO-FFI (Magalhães et al., 2014), construída a partir da versão Portuguesa do NEO-PI-R (Lima \& Simões, 1997; 2006). Tal como na versão original Norte Americana, esta versão reduzida é constituída por 60 itens (12 por dimensão), respondidos numa escala de likert de 5 pontos de 0 (discordo fortemente) a 4 (concordo fortemente). 0 tempo de preenchimento é de aproximadamente 15 minutos. A presente versão apresenta valores de consistência interna adequados: Conscienciosidade (.81), Neuroticismo (.81), Extroversão (.75), Amabilidade (.72) e Abertura à Experiência (.71) (Magalhães et al., 2014).

\section{Procedimentos}

A participação no presente estudo foi voluntária (a amostra foi recolhida através do método não probabilístico 'bola de neve') e a confidencialidade dos dados foi garantida. Os objetivos foram apresentados previamente aos participantes e estes referiram compreendê-los e aceitaram participar. Foi utilizado o IBM SPSS Statistics v. 21 para analisar os dados. No que diz respeito à normalidade da distribuição, os itens apresentaram todos valores absolutos de assimetria e de curtose abaixo de 3.0 e de 8.0, respetivamente (Kline, 2005).

Foram analisados os dados do ponto de vista descritivo e multivariado (MANCOVA) no sentido de apresentar os valores médios e desvio padrão das pontuações obtidas pelos sujeitos assim como o de testar a relação entre sexo e escolaridade e os cinco fatores de personalidade, controlando a idade.

\section{RESULTADOS}

\section{Análises descritivas}

Foram analisadas as médias e respetivo desvio padrão relativamente às cinco dimensões de personalidade, atentando à amostra global e às características sociodemográficas dos participantes, especificamente sexo, idade e escolaridade. Considerando a amostra global, podemos verificar que os participantes apresentam pontuações mais elevadas na dimensão de Conscienciosidade e mais reduzidas na dimensão de Neuroticismo (Quadro 1).

Quadro 1. Pontuações nas cinco dimensões de personalidade - amostra global ( $\mathrm{N}=1178)$.

\begin{tabular}{lcccc}
\hline & Mínimo & Máximo & Média & Desvio Padrão \\
\hline Neuroticismo & 0.00 & 48 & 23.92 & 7.46 \\
Extroversão & 7.00 & 44 & 29.55 & 6.01 \\
Abertura à Experiência & 5.00 & 46 & 27.54 & 6.30 \\
Amabilidade & 8.00 & 48 & 32.49 & 5.61 \\
Conscienciosidade & 4.00 & 48 & 34.26 & 6.31 \\
\hline
\end{tabular}


Este resultado pode estar relacionado com o facto de estarmos a reportar dados relativos a uma amostra mais escolarizada que a população Portuguesa em geral (levando a um aumento da Conscienciosidade), e não clínica (logo sendo de esperar um Neuroticismo mais baixo).

Quando analisamos os dados meramente descritivos das cinco dimensões de personalidade em função do sexo do participantes (Quadro 2), verificamos que o padrão observado com a amostra total se confirma no grupo do sexo masculino e feminino, sendo, no entanto, de salientar que o valor mais reduzido ao nível do Neuroticismo se observa no grupo do sexo masculino, e o valor mais elevado ao nível da Conscienciosidade foi observado no grupo do sexo feminino.

Quadro 2. Pontuações nas cinco dimensões de personalidade em função do sexo dos participantes.

\begin{tabular}{|c|c|c|c|c|}
\hline & \multicolumn{2}{|c|}{ Feminino $(\mathrm{N}=801)$} & \multicolumn{2}{|c|}{ Masculino $(\mathrm{N}=377)$} \\
\hline & Média & Desvio Padrão & Média & Desvio Padrão \\
\hline Neuroticismo & 25.02 & 7.10 & 21.59 & 7.68 \\
\hline Extroversão & 29.72 & 5.82 & 29.19 & 6.41 \\
\hline Abertura à Experiência & 27.74 & 6.16 & 27.11 & 6.59 \\
\hline Amabilidade & 32.97 & 5.42 & 31.48 & 5.86 \\
\hline Conscienciosidade & 34.51 & 6.20 & 33.72 & 6.51 \\
\hline
\end{tabular}

No que diz respeito às médias obtidas em função da idade dos participantes (Quadro 3) observamos que a Conscienciosidade continua a ser a dimensão com uma média superior nos grupos etários de sujeitos mais velhos, sendo que nos mais novos essa média passa a ser na dimensão da Amabilidade (apesar de muito próximas em termos absolutos). Já no que se refere à dimensão do Neuroticismo, esta mantém-se a dimensão com a média mais reduzida nos grupos até aos 64 anos, passando a ser a Abertura à Experiência a dimensão menos pontuada nos participantes a partir dos 65 anos.

Quadro 3. Pontuações nas cinco dimensões de personalidade em função da idade dos participantes.

\begin{tabular}{lccccc}
\hline & \multicolumn{2}{c}{$\begin{array}{c}\text { 18-24 anos } \\
\text { (N=444) }\end{array}$} & \multicolumn{2}{c}{$\begin{array}{c}\text { 25-64 anos } \\
\text { (N=486) }\end{array}$} & \multicolumn{2}{c}{$\begin{array}{c}\text { 65 mais anos } \\
\text { (N=248) } \\
\end{array}$} & Média & Desvio Padrão & Média & Desvio Padrão & Média & Desvio Padrão \\
\hline Neuroticismo & 24.26 & 7.74 & 23.24 & 7.53 & 24.66 \\
Extroversão & 30.97 & 5.46 & 29.67 & 5.97 & 26.77 \\
Abertura à Experiência & 30.09 & 5.50 & 27.62 & 5.98 & 6.11 \\
Amabilidade & 33.24 & 5.48 & 31.99 & 5.27 & 32.81 \\
Conscienciosidade & 33.17 & 6.39 & 35.26 & 6.17 & 34.23 \\
\hline
\end{tabular}

Finalmente, analisando os resultados descritivos obtidos em função da escolaridade (Quadro 4) verificamos o mesmo padrão ao nível da Conscienciosidade, com as médias mais elevadas em todos os grupos de escolaridade, e o Neuroticismo como sendo aquela dimensão que apresenta a média mais reduzida.

Quadro 4. Pontuações nas cinco dimensões de personalidade em função da escolaridade dos participantes.

\begin{tabular}{|c|c|c|c|c|c|c|}
\hline & \multicolumn{2}{|c|}{$\begin{array}{c}\text { Até ao } 3 \text { o Ciclo } \\
(\mathrm{N}=447)\end{array}$} & \multicolumn{2}{|c|}{$\begin{array}{l}\text { Ensino Secundário } \\
(\mathrm{N}=526)\end{array}$} & \multicolumn{2}{|c|}{$\begin{array}{c}\text { Ensino Superior } \\
(\mathrm{N}=205)\end{array}$} \\
\hline & Média & Desvio Padrão & Média & Desvio Padrão & Média & Desvio Padrão \\
\hline Neuroticismo & 24.06 & 7.05 & 24.25 & 7.80 & 22.78 & 7.37 \\
\hline Extroversão & 27.98 & 6.15 & 30.68 & 5.66 & 30.07 & 5.88 \\
\hline Abertura à Experiência & 24.07 & 5.69 & 29.63 & 5.72 & 29.75 & 5.62 \\
\hline Amabilidade & 32.04 & 5.77 & 32.96 & 5.51 & 32.28 & 5.41 \\
\hline Conscienciosidade & 34.90 & 6.41 & 33.35 & 6.30 & 35.17 & 5.80 \\
\hline
\end{tabular}

\section{Análises multivariadas}

Foram realizadas análises multivariadas no sentido de identificar diferenças de sexo e escolaridade em função das cinco dimensões de personalidade, controlando a variável idade. Assim, foram inicialmente testados os pressupostos para a realização da MANCOVA: a homogeneidade das Matrizes de Covariância foi testada através teste $M$ de Box $(p=.007)$ e a homogeneidade de variâncias através do teste de Levene (Neuroticismo: $p=.022$; Extroversão: $p=.074$; Abertura à Experiência: $p=.482$; Amabilidade: $p=.389$; Conscienciosidade: $p=.838$ ). A homogeneidade de variâncias foi assegurada através de valores de p nãosignificativos, com exceção do Neuroticismo. A multicolinearidade e a singularidade foram testadas pela Matriz Residual dos SSCP (sum of squares and cross product) e foram, neste estudo, asseguradas, com correlações entre os cinco fatores inferiores a 80 .

Os efeitos de interação das variáveis sexo e escolaridade não se revelaram significativos, $F(10$, $2336)<1$, mas detetamos efeitos principais significativos do $\operatorname{sexo} F(5,1167)=20.0, p<.001 ; \eta^{2}{ }_{p}=0.08$, 
$90 \%$ CI $[.053, .101]$ e da escolaridade $F(10,2336)=7.50, p<.001 ; \eta^{2} p=0.03,90 \%$ CI $[.017, .040]$ nas dimensões da personalidade, controlando a idade. Aliás, numa perspetiva multivariada, a variável concomitante idade revelou-se significativa $F(5,1167)=19.2, p<.001 ; \eta^{2} p=0.08,90 \%$ CI $[.051, .098]$. Na análise por dimensão de personalidade sendo estes efeitos verificados de forma significativa apenas na Extroversão $F(1,1171)=42.1, p<.001 ; \eta^{2} p=0.04,90 \%$ CI $[.020, .054]$ e na Abertura à Experiência $F(1$, $1171)=69.1, p<.001 ; \eta^{2} p=0.06,90 \%$ CI [.036, .078]. De uma forma geral, verificámos que à medida que a idade avança, diminuem as pontuações de Extroversão e de Abertura à Experiência, sendo, então os mais novos que apresentam valores mais altos nestas duas dimensões.

Foi, ainda, possível verificar que os participantes com escolaridade até ao $3^{\text {o }}$ Ciclo pontuam significativamente mais baixo na dimensão da Abertura à Experiência $F(2,1171)=29.22, \mathrm{p}<.001 ; \eta^{2} p$ $=.048,90 \%$ CI $[.029, .068]$ do que aqueles com o Ensino Secundário ou Superior; do mesmo modo, os participantes com Ensino Secundário apresentam uma pontuação superior na Conscienciosidade quando comparados com os dois outros grupos $F(2,1171)=5.68, p<.01 ; \eta^{2} p=.010,90 \%$ CI [.002, .020]. Quando analisamos as diferenças de sexo, verificamos que comparativamente aos homens, as mulheres pontuaram significativamente mais no Neuroticismo $F(1,1171)=49.65, p<.001 ; \eta^{2} p=.041,90 \%$ CI $[.024$, $.061]$, Conscienciosidade $F(1,1171)=6.87, p<.01 ; \eta^{2}{ }_{p}=0.06,90 \%$ CI $[.001, .015]$ e Amabilidade $F(1$, 1171) $=14.92, p<.001 ; \eta^{2} p=0.13,90 \%$ CI $[.004, .025]$.

\section{DISCUSSÃO}

Com o objetivo de tornar o NEO-FFI disponível para ser usado na avaliação da personalidade e na investigação, neste trabalho apresentam-se dados complementares, aos inicialmente levados a cabo por Magalhães e colaboradores (2014), com esta versão reduzida do NEO-PI-R. Os resultados da Análise em Componentes Principais e da Análise Factorial Confirmatória neste estudo prévio mostraram que a versão Portuguesa do NEO-FFI replica a estrutura de cinco fatores de forma congruente com os estudos transculturais disponíveis (e.g., Grã Bretanha, Espanha e Austrália) (Magalhães et al., 2014; McCrae \& Costa, 2004). A análise da fiabilidade da versão Portuguesa do NEO-FFI revelou ainda que o Neuroticismo e a Conscienciosidade foram as dimensões mais robustas, o que é congruente com a investigação internacional (ver Anexo 1).

Os próprios autores do teste (Costa \& McCrae, 1992) advertem que apesar do NEO-PI-R se ter mostrado adequado com diferentes amostras, e as normas serem baseadas na idade e no sexo do sujeito e na forma de administração do teste, correlações do inventário com variáveis, como a idade, sexo e anos de escolaridade, devem ser examinadas para analisar a influência que estas possam ter nas pontuações do teste. Quando estas correlações são elevadas, pode-se colocar a hipótese de que as influências demográficas estão a confundir as relações entre as pontuações dos traços e outros critérios.

Assim, neste estudo, ao analisar a relação entre a pontuação obtida pelos sujeitos nas cinco dimensões de personalidade e algumas das suas características individuais, verificámos que os participantes com menor escolaridade tendem a apresentar pontuações mais reduzidas na dimensão Abertura à Experiência. Este resultado é espectável visto que na sua própria definição conceptual este é o único fator relacionado com a criatividade que é uma dimensão da inteligência. Efetivamente, os sujeitos que pontuam de forma elevada na Abertura à Experiência tendem a procurar ativamente o conhecimento, e tal poderá ser estimulado ou reforçado de forma preferencial em níveis mais elevados de escolaridade. Por outro lado, quem é aberto à experiência terá mais facilidade em progredir academicamente visto, tendencialmente, ter uma criatividade/inteligência também maior. Do mesmo modo, aqueles participantes que apresentam níveis de escolaridade de grau intermédio (Ensino Secundário) ou superior apresentam pontuações de Conscienciosidade superiores.

No que diz respeito às diferenças de sexo, verificamos que as mulheres pontuaram significativamente mais no Neuroticismo, Amabilidade e Conscienciosidade. Estas diferenças são teoricamente plausíveis, uma vez que parece ser claro na literatura (Costa, Terracciano \& McCrae, 2001; McCrae, 2002) que não só há diferenças de sexo ao nível emocional (níveis mais elevados de ansiedade e nervosismo nas mulheres), como também uma expectativa social relacionada com o comportamento congruente com traços de amabilidade mais focados no sexo feminino (Costa, Terracciano, \& McCrae, 2001; Aluja, Garcia, Rossier, \& Garcia, 2005). No que concerne à Conscienciosidade elevada, esta pode explicar a maior sensatez e responsabilidade atribuída no presente ao género feminino, bem como, a maior longevidade feminina. Efetivamente, a literatura valoriza as pontuações na dimensão Neuroticismo e Conscienciosidade ou Amabilidade como fatores predictores ou protetores de declínio cognitivo (Chapman, Lyness \& Duberstein, 2007; Archer et al., 2006). Dados do Personality and Longevity Study from the Georgia centenarian study (Martin et al., 2006) sugerem que os centenários têm um Neuroticismo relativamente baixo e os outros quatro fatores elevados. 
Estes resultados justificam aliás as normas distintas em função do género e, também, da idade. No que concerne a esta última variável atributo de uma forma geral, verificámos que à medida que a idade avança diminuem as pontuações de Extroversão e de Abertura à Experiência, sendo, então, os mais novos que apresentam valores mais altos nestas duas dimensões.

A literatura inicial relativa ao NEO-PI-R e NEO-FFI refere a ausência de diferenças em função da idade - a estabilidade da personalidade na idade adulta. Apesar dos acontecimentos de vida adversos parecerem não ter impacto na personalidade dos indivíduos, investigação recente e meta-análises de estudos antigos indicam, no entanto, que as mudanças ocorrem nas cinco dimensões em diferentes momentos do ciclo de vida. A saber, Amabilidade e a Conscienciosidade tipicamente tendem a aumentar ao longo do tempo enquanto a Extroversão e o Neuroticismo e a Abertura a decrescer. Resultados aliás confirmados, em grande medida, na nossa investigação (Martin et al., 2006).

Apesar da importância e pertinência da utilização de uma versão breve do NEO-PI-R, importa salientar algumas limitações do presente estudo. De facto, a amostra aqui descrita é de conveniência e muitos dos participantes são jovens universitários do sexo feminino, o que poderá condicionar a análise e interpretação dos resultados aqui apresentados. Não obstante, estes dados reforçam as potencialidades da versão breve do NEO-PI-R para ser utilizada no contexto português, uma vez que são consistentes com o esperado do ponto de vista teórico. Para maior clarificação dos resultados e para a edificação das normas portuguesas do NEO-FFI seria importante realizar um estudo futuro com uma amostra representativa da nossa população - ou seja, com uma distribuição adequada dos sujeitos pelos diferentes grupos de idade, escolaridade e género. Apesar de alguns estudos, inclusivamente nacionais (e.g., Bertoquini \& Pais-Ribeiro, 2006) terem levado a cabo estudos comparativos com várias versões reduzidas seria importante reforçar estes estudos para aferir das eventuais vantagens ou, não do, uso do NEO-FFI comparativamente a outras medidas reduzidas ou breves dos cinco fatores (e.g., Mini-IPIP).

Em suma, os resultados obtidos corroboram a existência das comunalidades transculturais da personalidade inclusivamente ao nível das variáveis idade, género e escolaridade tornando mais claro o potencial desta "ferramenta de avaliação da personalidade" para a intervenção prática e aplicada (e.g., Magalhães, Costa, \& Costa, 2014). Os resultados deste estudo contribuem para considerar este instrumento como uma ferramenta necessária e valiosa no estudo da personalidade, e para que a versão Portuguesa do NEO-FFI possa ser vista como uma versão fiável do NEO-PI-R na representação das dimensões básicas da personalidade.

\section{Referências}

Aluja, A., Garcia, L. F., Cuevas, L., \& Garcia, O. (2007). The MCMI-III personality disorders scores predicted by NEO-FFI. Journal of Personality Disorders, 21, 58-71.

Aluja, A., Garcia, O., Rossier, J., \& Garcia, L. (2005). Comparison of the NEO-FFI, NEO-FFI-R and an alternative short version of the NEO-PI-R (NEO-60) in Swiss and Spanish samples. Personality and Individual Differences, 38(1), 591-604.

Archer, N., Brown, R. G., Boothby, H., Foy, C., Nicholas, H., \& Lovestone, S. (2006). The NEO-FFI is a reliable measure of premorbid personality in patients with probable Alzheimer's disease. International Journal of Geriatric Psychiatry, 21(5), 477-484.

Barros, A. (1979). Os valores e o modelo dos cinco factores da personalidade: Aplicação de dois instrumentos de medida a uma amostra de adultos trabalhadores. Dissertação de Mestrado apresentada à FPCE da Universidade de Lisboa.

Bertoquini, V., \& Pais-Ribeiro, J. L. (2006). Estudo de Formas Reduzidas do NEO-PI-R. Psicologia: Teoria, Investigação e Prática, 11(1), 85-101.

Borkenau, P., \& Ostendorf, F. (1993). NEO-Funf-Faktoren Inventar (NEO-FFI) nach Costa und McCrae: Handanweisung. Gottingen: Hogrefe.

Chamorro-Premuzic, T., \& Furham, A. F. (2009). Mainly Openness: The relationship between the Big Five personality traits and learning approaches. Learning and Individual Differences, 19, 524-529.

Chamorro-Premuzic, T., Quiroga, M. A., \& Colom, R. (2009). Intellectual competence and academic performance: A Spanish study. Learning and Individual Differences, 19, 486-491.

Chapman, B. P., Lyness, J. M., \& Duberstein, P. (2007). Personality and Medical Illness Burden Among Older Adults in Primary Care. Psychosomatic Medicine, 69(3), 277-282.

Costa, P. T., \& McCrae, R. R. (1988). The NEO-PI/FFI Manual Supplement. Odessa, FL: Psychological Assessment Resources.

Costa P. T., \& McCrae, R. R. (1989). The NEO PI Manual Supplement. Odessa, FL: Psychological Assessment Resources.

Costa, P. T., \& McCrae, R. R. (1992). The Revised NEO-PI/NEO-FFI Professional Manual. Odessa, FL: Psychological Assessment Resources. 
Costa, P. T., McCrae, R. R., \& Dye, D. A. (1991). Facet scales for agreeableness and conscientiousness: A revision of the NEO personality inventory. Personality and Individual Differences, 12(9), 887-898.

Costa, P. T., Terracciano, A., \& McCrae, R. R. (2001). Gender differences in personality traits across cultures: Robust and surprising findings - all 4 versions. Journal of Personality and Social Psychology, 81(2), 322-331.

Egan, V., Deary, I., \& Austin, E. (2000). The NEO-FFI: Emerging British norms and an item-level analysis suggest N, A, and C are more reliable than 0 and E. Personality and Individual differences, 29(5), 907-920.

Field, A. (2009). Discovering Statistics Using SPSS (2nd Edition). London: SAGE Publications Ltd.

Furham, A. F. (1997). Knowing and Faking One's Five-Factor Personality Score. Journal of Personality Assessment, 69(1), 229-243.

Gignac, G., Bates, T., \& Jang, K. (2007). Implications relevant to CFA model misfit, reliability, and fivefactor model as measured by the NEO-FFI. Personality and Individual Differences, 43(5), 1051-1062.

Hendricks, A., Perugini, M., \& Angleitner, A. et al. (2003). The Five-Factor Personality Inventory: CrossCultural Generalizability across 13 countries. European Journal of Personality, 17(5), 347-373.

Hojat, M., \& Xu, G. (2004). A visitor's guide to effect sizes: statistical significance versus practical (clinical) importance of research findings. Advances in Health Sciences Education Theory and Practice, 9(3), 241-249.

Holden, R. R., \& Fekken, G. C. (1992).The NEO Five-Factor Inventory in Canadian context: Psychometric properties for a sample of university women. Personality and Individual differences, 17(3), 441-444.

Hrebícková, M., Urbánek, T., Cermák, I., Szarota, P., Ficková, E., \& Orlická, L. (2002). The NEO Five-Factor Inventory in Czech, Polish, and Slovak contexts. In R. R. McCrae, \& J. Allik (Eds.), The Five-Factor Model of Personality across cultures (pp. 53-78). EUA: Series Kluwer.

Hu, L. \& Bentler, P. M. (1999). Cutoff criteria for fit indexes in covariance structure analysis: conventional criteria versus new alternatives. Structural Equation Modeling, 6(1), 1-55.

Kline, R. B. (2005). Principles and practice of structural equations modeling (2nd ed). London: Guilford Press.

Korner, A., Geyer, M., Roth, M., Drapeau, M., Schmutzer, G., Albani, C., Schumann, S., \& Brahler, E. (2008). Personality assessment with the NEO-Five-Factor Inventory: The 30-Item-Short-Version (NEO-FFI30). Psychotherapie Psychosomatik Medizinische Psychologie, 58(6), 238-245.

Lima, M. P. (1997). NEO-PI-R Contextos teóricos e psicométricos. Tese de doutoramento. Coimbra: FPCEUC.

Lima, M. P., \& Simões, A. (1997). O Inventário da Personalidade NEO-PI-R: Resultados da Aferição Portuguesa. Psychologica, 18, 25-46.

Lima, M. P. \& Simões, A. (2000). NEO-PI-R Manual Profissional (1ํㅡㄹ ed.). Lisboa: CEGOC.

Lima, M. P. \& Simões, A. (2006). Inventário de Personalidade NEO revisto (NEO-PI-R). In M. Gonçalves, L. Almeida, M. Simões, \& C. Machado (Eds.), Avaliação Psicológica - Instrumentos validados para a População Portuguesa (Vol. I) (2 ${ }^{\mathrm{a}}$ ed.) (pp. 15-32). Coimbra: Quarteto.

Lima, M. P. (2008). NEO-FFI - Versão Portuguesa. Poster apresentado na XIII

ConferênciaInternacional: Avaliação Psicológica "Formas e contextos" (Universidade do Minho, 2 a 4 de Outubro de 2008).

Little, B. R., \& Joseph, M. F. (2007). Personal projects and free traits: Mutable selves and well-beings. In B. R. Little, K. Salmela-Aro, \& S. D. Phillips. Personal Projects Pursuit: Goals, Action, and Human Flourishing (pp. 375- 400). New Jersey: Lawrence Erlbaum Associates Publishers.

Lucas, R. E., \& Donnellan, M. B. (2009). Age differences in personality: Evidence from a nationally representative Australian sample. Developmental Psychology, 45(5), 1353-63.

Magalhães, E.; Lima, M. P.; Salgueira, A.; Gonzalez, A.; Costa, J.J.; Costa, M.J.; Costa, P. (2014). NEO-FFI: Psychometric properties of a short personality inventory in a Portuguese contexto. Psicologia: Reflexão e Crítica. 27(4), 0-0.

Martin, P., da Rosa, G., Siegler, I. C., Davey, A., MacDonald, M., \& Poon, L. W. (2006). Personality and longevity: findings from the Georgia centenarian study. $A G E, 28(4), 343-352$.

Martinsen, Ø., Nordvik, H., \& Østbø. L. E. (2003). Norsk utgave av Revised NEO Personality Inventory (NEO PI-R). Oslo: Gyldendal Akademisk.

McAdams D. P., \& Olson, B. D. (2010). Personality development: Continuity and change over the life course. Annual Review of Psychology, 61, 517-542.

McAdams, D. P., \& Pals, J. L. (2006). A new big five: Fundamental principles for an integrative science of personality. American Psychologist, 61(3), 204-217. 
McCrae, R.R. (2002). NEO-PI-R data from 36 cultures: Further intercultural comparisons. In: McCrae, R.R.; Allik, J., editors. The Five-Factor Model of personality across cultures. New York: Kluwer Academic/Plenum Publishers; 105-125.

McCrae R. R., \& Costa P. T. (2004). A contemplated revision of the NEO five-factor inventory. Personality and Individual Differences, 36(3), 587-596.

McCrae R. R., Terracciano A., \& 78 Members of the Personality Profiles of Cultures Project (2005). Universal features of personality traits from the observer's perspective: Data from 50 cultures. Journal of Personality and Social Psychology, 88(3), 547-561.

McCrae, R. R., \& Costa, P. T., Jr. (2008). Empirical and Theoretical Status of the Five-Factor Model of Personality Traits. In G. J. Boyle, G. Matthews, \& D. H. Saklofske. The SAGE Handbook of Personality Theory and Assessment, (Vol. 1), (pp. 273-293). London: Sage Publications.

McCrae, R.R, Costa P. T. Jr, \& Martin T. A. (2005). The NEO-PI-3: A more readable Revised NEO Personality Inventory. Journal of Personality Assessment, 84:261-270.

McCrae, R., Costa, P. T., Lima, M. P., Simões, A., Ostendorf, F., Angleitner, A., Marusic, I., Bratko, D., Caprara, G. V., Barbaranelli, C., Chae, J. H., \& Piedmont, R. L. (1999). Age Differences in Personality Across the Adult Life Span: Parallels in Five Cultures. Developmental Psychology, 35(2), 466-477.

McCrae, R., Costa, P., del Pilar, G. H., Rolland, J. P., \& Parker, W. D. (1998). Cross-Cultural Assessment of the Five-Factor Model: The Revised NEO Personality Inventory. Journal of Cross-Cultural Psychology, 29, 171-188.

McCrae, R. R. \& Costa, P. T., Jr. (2003). Personality in Adulthood: A Five-Factor Theory Perspective (2nd ed.). New York: Guilford Press.

Nyater, T. E., Langvik, E., Berthelsen, M., \& Nordvik H. (2009). Interpersonal problems and personality traits: The relation between IIP-64C and NEO-FFI. Nordic Psychology, 61(3), 82-93.

Panayiotou, G., Kokkinosb, C.M., \& Spanoudisa, G. (2004). Searching for the 'Big five' in a Greek context: The NEO-FFI under the microscope. Personality and Individual Differences, 36(8), 1841-1854.

Rolland, J. P., Parker, W. D., \& Stumpf, H. (1998). A Psychometric Examination of the French Translations of the NEO-PI-R and NEO-FFI. Journal of Personality Assessment, 71(2), 269-291.

Satterwhite, R. C., Fogle, E. E., \& Williams, J. E. (1999). Revisiting the stability of variability: Traitedness and supertraitedness on the ACL and NEO-FFI. Social Behavior and Personality, 27(2), 205-220.

Schermelleh-Engel, K., Moosbrugger, H., \& Muller, H. (2003). Evaluating the fit of structural equation models: Tests of significance and descriptive goodness-of-fit measures. Methods of Psychological Research Online, 8(2), 23-74.

Schmitz, N., Hartkamp, N., Baldini, C., Rollnik, J., \& Tress, W. (2001). Psychometric properties of the German version of the NEO-FFI in psychosomatic outpatients. Personality and Individual Differences, 31(5), 713-722.

Yoshimura, K., Ono, K., Nakamura, J. H., Nathan \& K. Suzuki (2001). Validation of the Japanese version of the NEO Five-Factor Inventory in a large community sample. Psychological Reports, 88(2), 443449.

Historial do artigo

Recebido 22/03/2014

Aceite $\quad 11 / 09 / 2014$

Publicado 12/2014 
Anexo 1. Dados de validação do NEO-FFI em diferentes culturas.

\begin{tabular}{|c|c|c|c|c|c|c|c|}
\hline País & Autores & Amostra & Alfa N & Alfa E & Alfa 0 & Alfa A & Alfa C \\
\hline \multirow[t]{3}{*}{ USA } & Costa \& McCrae (1988/89; 1992) & - Amostra Normativa & .86 & .77 & .51 & .69 & .83 \\
\hline & & $\begin{array}{l}\text { - Amostras de estudantes } \\
\text { universitários } \mathrm{N}=1959\end{array}$ & .82 & .78 & .70 & .72 & .81 \\
\hline & McCrae \& Costa (2004) & $\begin{array}{l}\text { - Amostra do estudo } \\
\text { Longitudinal sobre o } \\
\text { envelhecimento de } \\
\text { Baltimore } \mathrm{N}=1492\end{array}$ & .86 & .80 & .75 & .69 & .79 \\
\hline Canadá & Holden \& Fekken (1992) & $\begin{array}{l}\text { Amostra de estudantes } \\
\text { universitários } \\
\mathrm{N}=243\end{array}$ & .86 & & & .68 & \\
\hline \multirow[t]{2}{*}{ França } & Rolland, Parker and Stumpf (1998) & $\begin{array}{l}\text { Amostra de estudantes } \\
\text { universitários } \mathrm{N}=447 \\
\text { Amostra Francesa }\end{array}$ & .84 & .77 & .62 & .66 & .84 \\
\hline & & Amostra Militar N=268 & .79 & .66 & .50 & .57 & .84 \\
\hline UK & Egan et al. (2000) & $\begin{array}{l}\text { Amostras de agricultores, } \\
\text { médicos e clínicos } \\
\mathrm{N}=1025\end{array}$ & .65 & .58 & .30 & .62 & .83 \\
\hline \multirow[t]{2}{*}{ Alemanha } & $\begin{array}{l}\text { Borkenau \& Ostendorf (1993) } \\
\text { Schmitz et al. (2001) }\end{array}$ & $\begin{array}{l}\text { Amostra de Adultos: } \\
\mathrm{N}=2112\end{array}$ & .85 & .80 & .71 & .71 & .71 \\
\hline & & $\mathrm{N}=300$ & .84 & .79 & .66 & .68 & .79 \\
\hline Suiça & $\begin{array}{l}\text { Aluja Garcia, Rossier e Garcia } \\
(2005 ; 2007)\end{array}$ & $\begin{array}{l}\text { Amostra de Adultos } \\
\text { Voluntários } \\
\mathrm{N}=1090\end{array}$ & .85 & .74 & .71 & .71 & .83 \\
\hline Espanha & $\begin{array}{l}\text { Aluja, Garcia, Rossier e Garcia ( } 2004 \\
\text { ou7) }\end{array}$ & $\begin{array}{l}\text { Amostra de estudantes } \\
\text { não graduados } \\
N=1006\end{array}$ & .82 & .78 & .71 & 71 & .83 \\
\hline Polónia; República & Hrebícková et al. (1991, 2002); & $\mathrm{N}=350$ & & & .69 & & .84 \\
\hline da Eslováquia; & & $\mathrm{N}=516$ & & & .66 & & .83 \\
\hline República Checa & & $\mathrm{N}=945$ & & & .60 & & .84 \\
\hline Japão & Yoshimura et al. (2001) & $\begin{array}{l}\text { Amostra Rural } \\
\mathrm{N}=1,664\end{array}$ & .60 & & & & .77 \\
\hline Austrália & Lucas \& Donnellan (2009) & $\mathrm{N}=12.618$ ( 15 a 84 anos $)$ & .85 & & .74 & & \\
\hline Grécia & $\begin{array}{l}\text { Panayiotou, Kokkinos e Spanoudis } \\
\text { (2004) }\end{array}$ & $\mathrm{N}=1204$ & .78 & .70 & .51 & 69 & .83 \\
\hline Noruega & Nordvik et al. (2003) & $\begin{array}{l}\mathrm{N}=1717 \text { (mulheres) } \\
\mathrm{N}=1751 \text { (homens) }\end{array}$ & .92 & & & .86 & \\
\hline
\end{tabular}


A versão portuguesa do NEO-FFI 